PROCEEDINGS OF THE

AMERICAN MATHEMATICAL SOCIETY

Volume 129, Number 12, Pages 3593-3602

S 0002-9939(01)06229-3

Article electronically published on June 6, 2001

\title{
UNIQUENESS AND ASYMPTOTIC BEHAVIOUR FOR SOLUTIONS OF SEMILINEAR PROBLEMS WITH BOUNDARY BLOW-UP
}

\author{
J. GARCÍA-MELIÁN, R. LETELIER-ALBORNOZ, AND J. SABINA DE LIS
}

(Communicated by David S. Tartakoff)

\begin{abstract}
In this paper we prove uniqueness of positive solutions to logistic singular problems $-\Delta u=\lambda(x) u-a(x) u^{p}, u_{\mid \partial \Omega}=+\infty, p>1, a>0$ in $\Omega$, where the main feature is the fact that $a_{\mid \partial \Omega}=0$. More importantly, we provide exact asymptotic estimates describing, in the form of a two-term expansion, the blow-up rate for the solutions near $\partial \Omega$. This expansion involves both the distance function $d(x)=\operatorname{dist}(x, \partial \Omega)$ and the mean curvature $H$ of $\partial \Omega$.
\end{abstract}

\section{INTRODUCTION AND RESULTS}

This research originated with the recent paper 10] which contains an exhaustive study of positive solutions $u$ to the logistic problem:

$$
\left\{\begin{array}{cl}
-\Delta u=\lambda u-a(x) u^{p}, & x \in \Omega^{\prime}, \\
u=0, & x \in \partial \Omega^{\prime},
\end{array}\right.
$$

$p>1, \lambda>0$ a parameter, $\Omega^{\prime} \subset \mathbb{R}^{n}$ a bounded domain and where $a \in C\left(\bar{\Omega}^{\prime}\right)$ satisfies $a>0$ in a proper subdomain $\Omega \subset \bar{\Omega} \subset \Omega^{\prime}$ while $a=0$ in $\bar{\Omega}^{\prime} \backslash \Omega$. It turns out that positive solutions $u$ can only exist when $\lambda_{1}\left(\Omega^{\prime}\right)<\lambda<\lambda_{1}(\Omega)$ ( $\lambda_{1}$ being the first Dirichlet eigenvalue in the corresponding domain) being unique in that range. Moreover, the solution $u_{\lambda}$ to $(\mathrm{L})$ satisfies $u_{\lambda} \rightarrow \infty$ uniformly on of $\Omega^{\prime} \backslash \Omega$ when $\lambda \uparrow \lambda_{1}(\Omega)$ while $u_{\lambda} \rightarrow \underline{u}$ in $C^{2, \alpha}(\Omega)$ as $\lambda \uparrow \lambda_{1}(\Omega)$, for certain $0<\alpha<1$, where $\underline{u}$ is the minimal solution to the singular boundary value problem,

$$
\left\{\begin{array}{cl}
-\Delta u=\lambda(x) u-a(x) u^{p}, & x \in \Omega, \\
u=+\infty, & x \in \partial \Omega,
\end{array}\right.
$$

being $\lambda(x)=\lambda_{1}(\Omega)$ in this precise case. The boundary condition is understood as $u(x) \rightarrow+\infty$ when $d(x):=\operatorname{dist}(x, \partial \Omega) \rightarrow 0+$. Observe that $a>0$ in $\Omega$ while $a=0$ on $\partial \Omega$.

Our main objectives here are to show the uniqueness of positive solutions to $(\mathrm{P})$ together with producing an exact two-term asymptotic expansion of the solutions $u$

Received by the editors April 17, 2000.

2000 Mathematics Subject Classification. Primary 35J25; Secondary 35B40.

Key words and phrases. Boundary blow-up, uniqueness, sub and supersolutions, distance function.

This work was supported by DGES, project PB96-0621 (Spain) and grant FONDECYT No. 1000333 (Chile). 
to $(\mathrm{P})$ near the boundary, involving the distance $d$ together with the mean curvature $H$ of $\partial \Omega$. We also analyze a class of perturbations of $(\mathrm{P})$.

Singular boundary value problems such as $(\mathrm{P})$ go back to the pioneering work [5] on automorphic functions and the equation $-\Delta u=-e^{u}$ in the plane, and were later studied under the general form $-\Delta u=-f(u)$ in $n$-dimensional domains in [12, [18. The more subtle questions of blow-up rates near $\partial \Omega$ and uniqueness of solutions are the goals of more recent literature. The problem $-\Delta u=a(x) e^{u}$ plus $u_{\mid \partial \Omega}=+\infty$ is shown to exhibit a unique solution in a smooth domain $\Omega$ together with an estimate of the form $u=\log d^{-2}+v(d)$ in [14] (where $a(x) \geq a_{0}>0$ and $v=O(1)$ as $d \rightarrow 0$ ) and in [1] (where $a \equiv 1, v=o(1)$ as $d \rightarrow 0$ ).

The equation $-\Delta u=-a(x) u^{p}$ is first considered in [16] $\left(a \equiv 1, p=\frac{n+2}{n-2}\right)$, [13], [2], [3], 20] $\left(a \geq a_{0}>0, p \geq 3\right.$ in [13]) and [8, 9] where the $p$-Laplacian extension $-\Delta_{p} u=-a(x) u^{q}, q>p-1, a \geq a_{0}>0$, is studied. In all of them, uniqueness is achieved together with a blow-up estimate under the form $u \sim A d^{-\alpha}$ as $d \rightarrow 0+$, with $\alpha=\frac{2}{p-1}$ and $A=\left(2(p+1) /\left\{a(p-1)^{2}\right\}\right)^{1 /(p-1)}$.

Uniqueness and blow-up rates of $-\Delta u=-f(u)$ are treated in [15] and [4] for selective classes of nonlinearities extending $f=e^{u}$ and $f=u^{p}$, where the explosive solution at $x=0$ of $-u^{\prime \prime}=-f(u)$ is involved. For instance in [4] and the case of $-\Delta u=-u^{p}$ it is shown that $u=A d^{-\alpha}(1+O(d))$ as $d \rightarrow 0+$ allows the possible presence of a second explosive term in the expansion of $u$ when $\alpha>1$ $(p<3)$. An explicit expression for this second term has been recently found in 7] as $u=A d^{-\alpha}(1+B d+o(d)), B$ given as a function of the mean curvature $H$, provided that $1<p<3$ (in the radial case this term had been already computed in [4] for all $p>1$ ).

In this paper we will produce sharper results in two directions. First, uniqueness of solutions to $(\mathrm{P})$ and some of its perturbations is achieved by removing the condition $a>0$ up to the boundary (uniqueness was obtained in [17] under the assumption $a(x) \geq a_{0}>0$ in $\bar{\Omega}$, however considerably weakening the smoothness of $\Omega$ ). It should be emphasized that $a=0$ on $\partial \Omega$ is a natural restriction for $a$ inherited from the logistic problem (L)! Second, we are extending the scope of the expansion $u=A d^{-\alpha}(1+B d+o(d))$ both to cover the full range $p>1$ and the more general nonlinearities in $(\mathrm{P})$ and its perturbations.

Theorem 1. Let $\Omega \subset \mathbb{R}^{n}$ be a bounded $C^{k}$ domain, $k \geq 4$, and $\lambda, a \in C^{\alpha}(\bar{\Omega})$ such that $a>0$ in $\Omega$. Then the singular boundary value problem $(P)$ admits a minimal and a maximal classical solution. If moreover $a=0$ on $\partial \Omega$ and

$$
a(x)=C_{0} d^{\gamma}+o\left(d^{\gamma}\right)
$$

as $d \rightarrow 0+$ with $\gamma>0$ and $C_{0}>0$, then every solution $u \in C^{2}(\Omega)$ to $(P)$ satisfies

$$
\lim _{d \rightarrow 0+} \frac{u}{A d^{-\alpha}}=1
$$

where $\alpha=(\gamma+2) /(p-1)$ and $A=\left(\alpha(\alpha+1) / C_{0}\right)^{\frac{1}{p-1}}$. As a consequence, $(P)$ admits $a$ unique positive solution. In addition, if

$$
a(x)=C_{0} d^{\gamma}\left(1+C_{1} d+o(d)\right)
$$

as $d \rightarrow 0+$, a further estimate of the blow-up rate is available, namely

$$
u(x)=A d^{-\alpha}(1+B(s) d+o(d))
$$


as $d \rightarrow 0+$, where

$$
B(s)=\frac{(n-1) H(s)-(\alpha+1) C_{1}}{\gamma+p+3},
$$

with $H(s)$ standing for the mean curvature of $\partial \Omega$ in $s$, and for every $x$ near $\partial \Omega$, $d(x):=\operatorname{dist}(x, \partial \Omega)$ and $s=s(x)$ means the projection of $x$ over $\partial \Omega$.

Remark 1. a) The conclusions remain valid if the constants $C_{0}$ and $C_{1}$ are allowed to be functions of $s, C_{0}=C_{0}(s), C_{1}=C_{1}(s) \in C^{2}(\bar{\Omega})$ as long as $C_{0}(s)>0$ on $\partial \Omega$. Of course, the special case $\gamma=0$ in (1) and (3) falls within the scope of Theorem 1.

b) A special case of estimate (2) with $a$ vanishing at $\partial \Omega$ was obtained in [19].

c) Suppose more generally that $\partial \Omega$ splits into $\Gamma_{1}, \Gamma_{2}, \ldots, \Gamma_{m}$ connected pieces and consider the problem $-\Delta u=\lambda(x) u-a(x) u^{p}$ in $\Omega, u_{\mid \Gamma_{i}}=\sigma_{i}, 1 \leq i \leq m$, with $\sigma_{i} \geq 0$, some of them $+\infty$. Then the conclusions of Theorem 1 hold, since estimates (2) and (4) are understood near the connected components $\Gamma_{i}$ such that $\sigma_{i}=+\infty$.

Consider now the more general class of problems:

$$
\left\{\begin{array}{cl}
-\Delta u=\lambda(x) u-a(x)\left(u^{p}+g(x, u)\right), & x \in \Omega, \\
u=+\infty, & x \in \partial \Omega,
\end{array}\right.
$$

where $g$ is of lower order than $u^{p}$ at infinity. Then we have the following results:

Theorem 2. Let $\Omega \subset \mathbb{R}^{n}$ be a bounded $C^{k}$ domain, $k \geq 4$, and $\lambda, a \in C^{\alpha}(\bar{\Omega})$ such that $a>0$ in $\Omega$. If $g \in C(\bar{\Omega} \times \mathbb{R})$ is locally Lipschitz in $\bar{\Omega} \times \mathbb{R}$ and it satisfies the growth conditions

$$
\lim _{u \rightarrow 0} \frac{g(x, u)}{u}=0, \quad \lim _{u \rightarrow+\infty} \frac{g(x, u)}{u^{p}}=0
$$

uniformly in $x \in \bar{\Omega}$, then problem $(P)_{g}$ admits at least a classical solution $u \in$ $C^{2, \alpha}(\Omega)$. If a $(x)$ vanishes on $\partial \Omega$ and verifies (1), then estimate (2) holds for every possible solution to $(P)_{g}$. If, in addition, $g$ is continuously differentiable with respect to $u, g_{u}^{\prime} \in C(\bar{\Omega} \times \mathbb{R})$, while

$$
\lim _{u \rightarrow 0} \frac{g(x, u)}{u}=0, \quad \lim _{u \rightarrow+\infty} \frac{g_{u}^{\prime}(x, u)}{u^{p-1}}=0
$$

uniformly in $\bar{\Omega}$, then $(P)_{g}$ admits a unique positive solution, provided that $|a|_{\infty}$ is small enough.

Remark 2. a) The Lipschitz condition on $g$ can be relaxed to continuity if only strong or weak solutions instead of classical solutions are considered.

b) When $\lambda(x)>0$ in $\bar{\Omega}$, precise estimates on the size of $|a|_{\infty}$ in order to get uniqueness can be given solely in terms of $g$ and the geometry of $\Omega$ (see section 4 ).

c) If $a$ verifies (3) and $g$, together with its derivatives with respect to $u$ up to order three, satisfies suitable growth conditions, then every solution to $(\mathrm{P})_{g}$ admits in addition the asymptotic expansion (4).

d) The conclusions of Theorem 2 hold for the kind of perturbed equations $-\Delta u=$ $\lambda(x) u-a(x) u^{p}+a^{*}(x) g(x, u), g$ as above, $a^{*} \in C^{\alpha}(\bar{\Omega})$, but now with $\left|a^{*}\right|_{\infty}$ small. However, it is required that $a^{*}(x)=O(a(x))$ at $\partial \Omega$. Otherwise the problem could fall in the superlinear regime where even the existence of solutions may be lost. 
The next sections are dedicated to the proof of Theorems 1 and 2. Section 2 is concerned with existence and uniqueness of $(\mathrm{P})$. Estimate $(4)$ is obtained in section 3 while the analysis of the perturbed problem $(\mathrm{P})_{g}$ is carried out in section 4 .

\section{EXISTENCE AND UNIQUENESS}

Proof of the existence of solutions. Firstly, it should be remarked that positive solutions to the finite problem $-\Delta u=\lambda(x) u-a(x) u^{p}$ in $\Omega, u_{\mid \partial \Omega}=\sigma(x)$ are unique as a consequence of the general result in [6]. Now choose $\delta>0$ such that $p>1+\delta$, and consider the problem

$$
\left\{\begin{array}{cl}
-\Delta u=\lambda(x) u-\left(a(x)+\frac{1}{n^{\delta}}\right) u^{p}, & x \in \Omega, \\
u=n, & x \in \partial \Omega .
\end{array}\right.
$$

Since 0 is a subsolution and $n$ is a supersolution for $n$ large, $(\mathrm{P})_{n}$ admits a solution $u_{n} \in C^{2, \alpha}(\bar{\Omega})$ with $u_{n} \leq n$, which as quoted before is unique. Using [6], it can be shown that $\left\{u_{n}\right\}_{n}$ is increasing. Our purpose is to pass to the limit as $n \rightarrow \infty$ in $(\mathrm{P})_{n}$.

The validity of the following lemma (cf. [10]) is the key to guaranteeing the existence of the limit.

Lemma 3. Let $B \subset \mathbb{R}^{n}$ be an arbitrary ball and consider the problem

$$
\left\{\begin{array}{cl}
-\Delta u=\lambda u-A u^{p}, & x \in B, \\
u=\sigma, & x \in \partial B,
\end{array}\right.
$$

where $A, \lambda$ are positive constants and $\sigma>(\lambda / A)^{\frac{1}{p-1}}$. Then the unique solution $u_{\sigma, B}$ to (6) is a radial function, $u_{\sigma, B} \in C^{\infty}(\bar{B})$, while $u_{\sigma, B}$ converges as $\sigma \rightarrow+\infty$ to $\underline{u}_{B}$ in $C^{k}(B)$ for every $k \in \mathbb{N}$. Moreover, $\underline{u}_{B}$ defines the minimal solution to (6) with $\sigma=+\infty$. Finally, the following estimate holds:

$$
\inf _{B} \underline{u}_{B} \geq \frac{B_{0}^{2}}{2(p+1) A R^{2}}
$$

where $B_{0}=B\left(\frac{p-1}{2(p+1)}, \frac{1}{2}\right)$ and $B$ is the Euler Beta function.

Let us finish the proof of the existence assertion. If $u_{n}$ is the solution to $(\mathrm{P})_{n}$ we have $-\Delta u_{n} \leq \sup _{B} \lambda(x) u_{n}-\inf _{B} a(x) u_{n}^{p}$ in $B$. Since $u_{n} \leq n$ in $\bar{B}$, we obtain $u_{n}<u_{n, B}<\underline{u}_{B}$ in $B$, where $u_{n, B}$ is as in Lemma 3 . This together with the monotonicity of $\left\{u_{n}\right\}$ leads to $u_{n} \rightarrow \underline{u}, \underline{u} \in L_{\text {loc }}^{\infty}(\Omega)$. By elliptic estimates and a standard bootstrapping argument we achieve $u_{n} \rightarrow \underline{u}$ in $C^{2, \alpha}(\Omega)$.

To prove the minimal character of $\underline{u}$, let $u$ be an arbitrary solution to $(\mathrm{P})$. Since $u \geq n$ near $\partial \Omega$, for every positive integer $n$, an inner approximation argument leads to $u \geq u_{n}$, and consequently $u \geq \underline{u}$, as was to be proved.

The existence of a maximal solution follows a similar reasoning.

The following result is especially useful when dealing with the perturbed problem $(\mathrm{P})_{g}$. The proof, which is omitted for brevity, is carried out by applying the suband supersolutions method to problem $(\mathrm{P})$ in domains $\{x \in \Omega: d(x)>1 / n\}$, and doing $n \rightarrow \infty$ through a diagonal process. 
Lemma 4. Let $v, w \in C^{2}(\Omega)$ be functions such that $v(x) \leq w(x)$ in $\Omega,-\Delta v \leq$ $\lambda(x) v-a(x) v^{p},-\Delta w \geq \lambda(x) w-a(x) w^{p}$ in $\Omega$ and $\lim _{d(x) \rightarrow 0+} v(x)=\lim _{d(x) \rightarrow 0+} w(x)$ $=+\infty$. Then there exists at least a solution $u \in C^{2}(\Omega)$ of problem $(P)$ satisfying $v(x) \leq u(x) \leq w(x)$ in $\Omega$.

Remark 3. The features described in Lemma 4 also hold for the extended problem introduced in Remark $1 \mathrm{c}$ ).

Proof of estimate (2). We are only assuming that $a$ verifies hypothesis (1). Fix $\delta_{0}>0$ so that $d(x):=\operatorname{dist}(x, \partial \Omega)$ is a $C^{k}$ function in $0<d(x)<\delta_{0}$. Fixing an arbitrary $\varepsilon>0$, we have $\left(C_{0}-\varepsilon\right) d^{\gamma} \leq a(x) \leq\left(C_{0}+\varepsilon\right) d^{\gamma}$, as long as $0 \leq d<\delta_{1}$. Choosing

$$
A^{+}=\left\{\frac{\alpha(\alpha+1)}{C_{0}-2 \varepsilon}\right\}^{\frac{1}{p-1}}
$$

it is easy to prove that $u^{+}=A^{+} d^{-\alpha}$, defined for small $d>0$, is a supersolution. Indeed, it is enough to prove $-\Delta u^{+} \geq \lambda(x) u^{+}-\left(C_{0}-\varepsilon\right) d^{\gamma}\left(u^{+}\right)^{p}$ for $d \sim 0+$ $\left(d<\min \left\{\delta_{0}, \delta_{1}\right\}\right)$. Since $-\Delta u^{+}=-A^{+} \alpha(\alpha+1) d^{-\alpha-2}+O\left(d^{-\alpha-1}\right)$, this is a consequence of $\alpha(\alpha+1) \leq\left(C_{0}-\varepsilon\right)\left(A^{+}\right)^{p-1}+O(d)$, for $d$ small enough, which is in turn implied by the choice of $A^{+}$.

Symmetric calculations show that $u^{-}=A^{-} d^{-\alpha}, A^{-}=\left(\alpha(\alpha+1) /\left(C_{0}+2 \varepsilon\right)\right)^{1 /(p-1)}$, satisfies $-\Delta u^{-} \leq \lambda(x) u^{-}-\left(C_{0}+\varepsilon\right) d^{\gamma}\left(u^{-}\right)^{p}$ near $\partial \Omega$, therefore defining a local subsolution to $(\overline{\mathrm{P}})$ there.

Let us proceed now to estimate the blow-up rate of an arbitrary solution $u$ to (P), under the form (2).

Let us ascertain under which conditions $u^{+}+K$ turns out to be a supersolution near $\partial \Omega$. The function $\lambda(x) u-a(x) u^{p}$ is decreasing in $u$ when $\lambda(x) \leq 0$, or if $u>u_{c}(x):=\left(\frac{\left|\lambda^{+}\right|_{\infty}}{a(x)}\right)^{1 /(p-1)}$, with $\lambda^{+}=\max \{\lambda, 0\}$. Taking into account that

$$
u_{c}(x) \sim\left(\frac{\lambda}{C_{0}}\right)^{1 /(p-1)}\left(\frac{1}{d}\right)^{\frac{\gamma}{p-1}}, \quad \text { while } u^{+}(x) \sim A\left(\frac{1}{d}\right)^{\frac{\gamma+2}{p-1}},
$$

we can ensure that for small $d$ (say $0<d<\delta^{*}$ ), $u^{+}(x)>u_{c}(x)$ holds. Thus,

$$
-\Delta\left(u^{+}+K\right) \geq \lambda(x)\left(u^{+}+K\right)-a(x)\left(u^{+}+K\right)^{p},
$$

for $0<d<\delta^{*}, K>0$. Then

On the other hand, fix $0<\tau<\frac{\delta^{*}}{4}$ and introduce the region $Q_{\tau}:=\left\{\tau<d<\frac{\delta^{*}}{2}\right\}$.

$$
-\Delta\left(u^{+}(d-\tau, s)+K\right) \geq \lambda(x)\left(u^{+}(d-\tau, s)+K\right)-a(d, s)\left(u^{+}(d-\tau, s)+K\right)^{p}
$$

in $Q_{\tau}$, where the functions involved have been expressed in local coordinates $(d, s)$. Moreover, this fact is uniformly valid, no matter what the value of $\tau$ is.

Thus, for every $\tau \in\left(0, \frac{\delta^{*}}{4}\right), v^{+}=u^{+}(d-\tau, s)+K$ is a supersolution to

$$
\left\{\begin{array}{cl}
-\Delta v=\lambda(x) v-a(x) v^{p}, & x \in Q_{\tau}, \\
v=u, & x \in \partial Q_{\tau},
\end{array}\right.
$$

with $u$ an arbitrary fixed solution to $(\mathrm{P})$, provided that $K>0$ is chosen so that $u^{+}\left(\delta^{*} / 2-\tau, s\right)+K \geq u\left(\delta^{*} / 2, s\right)$, for every $0<\tau<\frac{\delta^{*}}{4}, s \in \partial \Omega$. In addition, 
the auxiliary problem (7) has $v=u$ as its unique solution. Since $v_{-}=0$ is a subsolution, we conclude

$$
u(x)=u(d, s) \leq u^{+}(d-\tau, s)+K,
$$

for every $s \in \partial \Omega, 0<\tau<d<\delta^{*} / 2$ and $0<\tau<\delta^{*} / 4$. Letting $\tau \rightarrow 0+$ we arrive at $u(x)=u(d, s) \leq u^{+}(x)+K$ in $d(x)<\delta^{*} / 2$, and we obtain

$$
\varlimsup_{d \rightarrow 0+} \frac{u(x)}{A d^{-\alpha}} \leq 1 .
$$

In what follows we will obtain the complementary estimate to (8). With an argument similar to the previous one, we conclude $u \geq \theta u^{-}$if $0<d(x)<\delta^{*} / 4$, for some $0<\theta<1$.

Our next objective is finding $\delta>0$ small enough, $\tau_{0}=\tau_{0}(\delta)$ and a constant $K=K(\delta)$ in such a way that $u^{-}(d+\tau, s)-K(\delta)$ defines a subsolution to $-\Delta u=$ $\lambda(x) u-a(x) u^{p}$ in the domain $\Omega_{\delta}:=\{0<d(x)<\delta\}$ for every $\tau \in\left(0, \tau_{0}(\delta)\right)$, also satisfying

$$
u^{-}(d+\tau, s)-K(\delta) \leq u(d, s)
$$

for $d=\delta$ and every $0<\tau<\tau_{0}(\delta)$. Inequality (9) is implied by $u^{-}(\delta+\tau, s)-$ $\theta u^{-}(\delta, s) \leq K(\delta)$, where $0<\tau<\tau_{0}(\delta)$. By virtue of the decreasing character of $u^{-}$with $d$, this last inequality is a consequence of

$$
K(\delta) \sim(1-\theta) A \delta^{-\alpha},
$$

as $\delta \rightarrow 0+$.

On the other hand, $u^{-}(d+\tau, s)-K(\delta)$ will be a subsolution if

$$
u^{-}(d+\tau, s)-K(\delta) \geq\left(\frac{\lambda}{p\left(C_{0}+\varepsilon\right)(d+\tau)^{\gamma}}\right)^{\frac{1}{p-1}},
$$

for $0<d+\tau<\delta+\tau_{0}(\delta)=(1+\zeta) \delta$ if $\tau_{0}(\delta)=\zeta \delta$ is chosen for some fixed $\zeta \in(0,1)$. For the validity of (10) it is enough to have

$$
A\left(\frac{1}{d+\tau}\right)^{\alpha}-(1-\theta) A\left(\frac{1}{\delta}\right)^{\alpha}>\left\{\frac{\lambda}{p C_{0}}\right\}^{\frac{1}{p-1}}\left(\frac{1}{d+\tau}\right)^{\frac{\gamma}{p-1}}
$$

for $0<d<(1+\zeta) \delta$, which in turn is true if $\zeta, \delta$ are small enough.

We therefore obtain that

$$
u^{-}(d+\tau, s)-K(\delta)
$$

defines for $0<\tau<(1+\zeta) \delta$ a family of finite subsolutions to problem $-\Delta v=$ $\lambda(x) v-a(x) v^{p}$ in $\Omega_{\delta}$, with $v=u$ in $d=\frac{\delta^{*}}{4}$ and $v=+\infty$ in $d=\delta$. Then $u^{-}(d+\tau, s)-K(\delta) \leq u(d, s)$ if $0<d<\delta$, and letting $\tau \rightarrow 0+$ we arrive at

$$
u^{-}(d, s)-K(\delta) \leq u(s, d), \quad 0<d<\delta,
$$

which completes the proof of (2).

Proof of uniqueness. Now let $u$ and $v$ be positive solutions to (P). By virtue of (2), $u$ and $v$ satisfy $\lim _{d(x) \rightarrow 0+} u(x) / v(x)=1$. Thus, for every $\varepsilon>0$, we can find $\delta>0$ (as small as we please) such that

$$
(1-\varepsilon) v(x) \leq u(x) \leq(1+\varepsilon) v(x)
$$


when $0<d(x) \leq \delta$. On the other hand, $w^{-}=(1-\varepsilon) v(x)$ and $w^{+}=(1+\varepsilon) v(x)$ are sub- and supersolutions to

$$
\left\{\begin{aligned}
-\Delta w=\lambda(x) w-a(x) w^{p}, & x \in \Omega \backslash \Omega_{\delta}, \\
w=u, & x \in \partial\left(\Omega \backslash \Omega_{\delta}\right),
\end{aligned}\right.
$$

where $\Omega_{\delta}=\{0<d(x)<\delta\}$. The unique solution to this problem is $w=u$. Then $(1-\varepsilon) v(x) \leq u(x) \leq(1+\varepsilon) v(x)$ holds in $\Omega \backslash \Omega_{\delta}$, and (11) is true in $\Omega$. Letting $\varepsilon \rightarrow 0+$ we arrive at $u=v$.

\section{Estimates FOR THE SECOND TERM}

We will now obtain estimate (4), and so we henceforth assume that the function $a$ verifies (3).

In this case, with an argument similar to the one used to show estimate (2), we can obtain for every $\varepsilon>0$ a pair of sub- and supersolutions of the form

$$
u^{ \pm}=A d^{-\alpha}\left(1+B^{ \pm} d\right), \quad 0<d(x)<\delta,
$$

where $B^{+} \geq B, B^{-} \leq B, B^{ \pm} \rightarrow B$ as $\varepsilon \rightarrow 0+, B$ given by (5). It is worthy of mention to say that $\Delta d(x) \rightarrow-(n-1) H(s)$ when $d(x) \rightarrow 0+$, where $H(s)$ is the mean curvature of $\partial \Omega$ at $s$ (cf. [11]).

Moreover, $u^{+}+K$ and $u^{-}-K(\delta)$ are still sub- and supersolutions with a convenient choice of $K$ and $K(\delta)$. However, this only leads to estimate (4) in the case $\alpha>1$, that is, for $p<\gamma+3$. This is why we need an alternative procedure for the complementary range $p \geq \gamma+3$. The following reasoning is indeed valid if $p \geq 2$.

We are looking for a constant $K>0$ such that

$$
-\Delta\left(A K d^{2-\alpha}\right) \geq \lambda(x) A K d^{2-\alpha}+a(x)\left(u^{+}\right)^{p}-a(x)\left(u^{+}+A K d^{2-\alpha}\right)^{p} .
$$

This inequality, together with $-\Delta u^{+} \geq \lambda(x) u^{+}-a(x)\left(u^{+}\right)^{p}$, will imply that $u^{+}+$ $A K d^{2-\alpha}$ is a supersolution to $(\mathrm{P})$ near $\partial \Omega$.

Since for $t \geq 0,1-(1+t)^{p} \leq-p t$, it is enough to prove $\Delta d^{2-\alpha} \leq-\lambda(x) d^{2-\alpha}+$ p $a(x)\left(u^{+}\right)^{p-1} d^{2-\alpha}$, which is independent of $K$. Now $\Delta d^{2-\alpha}=(2-\alpha)(1-\alpha) d^{-\alpha}+$ $O\left(d^{-\alpha+1}\right)$, and $a(x) \geq C_{0} d^{\gamma}(1-\varepsilon)$ for small $d$, so this is a consequence of

$$
(2-\alpha)(1-\alpha) \leq p \alpha(\alpha+1)(1-\varepsilon)+O(d),
$$

which in turn holds if $(2-\alpha)(1-\alpha)<p \alpha(\alpha+1)$. This inequality holds no matter what the values of $p$ and $\gamma$ are, thus we have a supersolution $v^{+}=u^{+}+A K d^{2-\alpha}$, for every $K>0$.

If $u$ is an arbitrary solution to (P) and we take $K$ to have $v^{+} \geq u$ in $d=\delta$, we obtain, with an argument similar to the one used in section 2 , that $v^{+} \geq u$ in $0<d(x)<\delta$. Thus letting $d \rightarrow 0+$ and then $\varepsilon \rightarrow 0+$, we conclude

$$
\varlimsup_{d \rightarrow 0+} \frac{1}{d}\left(\frac{u(x)}{A d^{-\alpha}}-1\right) \leq B .
$$

The lower estimate is not obtained in a completely similar way. We are now bound to find $K$ so that

$$
-\Delta\left(-A K d^{2-\alpha}\right) \leq-\lambda(x) A K d^{2-\alpha}+a(x)\left(u^{-}\right)^{p}-a(x)\left(u^{-}-A K d^{2-\alpha}\right)^{p},
$$

and we will get that $v^{-}=u^{-}-A K d^{2-\alpha}$ is a subsolution. 
If $p \geq 2, t \geq 0$, we have $1-(1-t)^{p} \geq p t-p(p-1) t^{2} / 2$, and it is then sufficient to show

$$
\Delta d^{2-\alpha} \leq p a(x)\left(u^{-}\right)^{p-1}\left(d^{2-\alpha}-\frac{p-1}{2} \frac{A K d^{4-2 \alpha}}{u^{-}}\right)-\lambda(x) d^{2-\alpha},
$$

which is implied by

$$
(2-\alpha)(1-\alpha) \leq p a(x) \frac{\alpha(\alpha+1)}{C_{0} d^{\gamma}}\left(1+B^{-} d\right)^{p-1}\left(1-\frac{p-1}{2} \frac{K d^{2}}{1+B^{-} d}\right)+O(d) .
$$

Since in addition

$$
\frac{K d^{2}}{1+B^{-} d} \leq \frac{K \delta^{2}}{1-\left|B^{-}\right|_{\infty} \delta},
$$

if we choose $K \delta^{2}=2 \theta\left(1-\left|B^{-}\right|_{\infty} \delta\right) /(p-1), 0<\theta<1$, it is enough to have

$$
(2-\alpha)(1-\alpha)<p \alpha(\alpha+1)(1-\varepsilon)(1-\theta) .
$$

We can certainly take $\varepsilon$ and $\theta$ small enough to satisfy (12). With this choice, $v^{-}=u^{-}-A K d^{2-\alpha}$ is a subsolution.

Now let $u$ be an arbitrary solution to $(\mathrm{P})$. We need to choose $K$ so that $v^{-} \leq u$ if $d=\delta$. Since $u \geq(1-\varepsilon) A d^{-\alpha}$, it suffices to have

$$
A \delta^{-\alpha}\left(1+B^{-} \delta\right)-A K \delta^{2-\alpha} \leq(1-\varepsilon) A \delta^{-\alpha} .
$$

This last inequality is a consequence of $\theta>(p-1) \varepsilon / 2$, which we can achieve by diminishing $\varepsilon$ if necessary.

To summarize, we arrive at $u \geq v^{-}$in $0<d<\delta$, which in turn implies

$$
\varliminf_{d \rightarrow 0+} \frac{1}{d}\left(\frac{u(x)}{A d^{-\alpha}}-1\right) \geq B,
$$

and estimate (4) is proved.

\section{Perturbed problems}

This section is devoted to the proof of Theorem 2. To begin with the existence notice that $(\mathrm{P})_{g}$ admits arbitrarily large supersolutions. Indeed, given $\varepsilon>0$, we have $g(x, u) \geq-\varepsilon u^{p}$ if $u \geq u_{0}(\varepsilon)>0$. So, denoting by $v$ the unique solution to the problem $-\Delta u=\lambda(x) u-(1-\varepsilon) a(x) u^{p}, u_{\partial \Omega}=+\infty$ and choosing $\theta>1$ to have $\theta v \geq u_{0}$, it turns out that $u^{+}=\theta v$ is a supersolution to $(\mathrm{P})_{g}$. On the other hand, it is possible to construct an increasing sequence $\left\{u_{n}\right\}, u=u_{n}$ a solution of:

$$
\left\{\begin{array}{cl}
-\Delta u=\lambda(x) u-a(x)\left(u^{p}+g(x, u)\right), & x \in \Omega, \\
u=n, & x \in \partial \Omega,
\end{array}\right.
$$

as follows: $u_{0}=0, u_{n+1}$ obtained using $u_{n}$ as a subsolution and $u^{+}$as a supersolution. The arguments in section 2 lead to $u_{n} \rightarrow u, u \in C^{2, \alpha}(\Omega)$ a solution to $(\mathrm{P})_{g}$.

To achieve uniqueness we will first prove estimate $(2)$ for all solutions to $(\mathrm{P})_{g}$. Accordingly, let $u$ be any solution to $(\mathrm{P})_{g}$ and choose $\varepsilon>0, u_{0}(\varepsilon)$ as above. Taking $\delta$ small to have $u \geq u_{0}$ in $\Omega_{\delta}=\{0<d(x)<\delta\}$, it follows that $u$ is a subsolution to

$$
\left\{\begin{array}{cl}
-\Delta v=\lambda(x) v-a(x)(1-\varepsilon) v^{p}, & x \in \Omega_{\delta}, \\
v=u, & x \in \partial \Omega_{\delta} .
\end{array}\right.
$$


It is easy to prove that $\theta u$ is a supersolution to (13) if $\theta$ is large. Since uniqueness holds for problem (13) (Remark 1 c)), then Lemma 4 and Remark 3 lead to

$$
\varlimsup_{d \rightarrow 0+} \frac{u}{A d^{-\alpha}} \leq(1-\varepsilon)^{-1 /(p-1)}
$$

Letting $\varepsilon \rightarrow 0$, we obtain $\varlimsup \lim u / A d^{-\alpha} \leq 1$. The lower estimate is performed in a similar way. Thus (2) is proved.

Now let us undertake the uniqueness assertion. Assume $g$ satisfies $(\mathrm{H})_{g^{\prime}}$. We claim the existence of $c>0$ such that every positive solution $u$ to $(\mathrm{P})_{g}$ verifies $u \geq c$ in $\Omega$. Thus, defining $M_{g}=\max \left\{0, \sup _{u \geq c} \frac{g(x, u)}{u^{p}}\right\}$, we conclude that every solution $u$ to $(\mathrm{P})_{g}$ satisfies $u \geq w$, where $w$ uniquely solves the problem

$$
\left\{\begin{array}{cl}
-\Delta w=\lambda(x) w-\left(1+M_{g}\right)|a|_{\infty} w^{p}, & x \in \Omega, \\
w=+\infty, & x \in \partial \Omega .
\end{array}\right.
$$

On the other hand, problem $(\mathrm{P})^{\prime}$ can be estimated from below by the one-dimensional version of $(6)$ on intervals $(-R, R)$, with $2 R \geq L_{\Omega}, L_{\Omega}$ being the minimum distance between parallel hyperplanes enclosing $\Omega$ (see [10] for more details). Hence, from Lemma 3 we have the estimate

$$
\inf _{\Omega} w \geq \frac{2 B_{0}^{2}}{(p+1)\left(1+M_{g}\right) L_{\Omega}^{2}|a|_{\infty}} .
$$

Thus if $|a|_{\infty}$ is small enough we have that all positive solutions $u$ to $(\mathrm{P})_{g}$ fall within the range where $u^{p-1}+g(x, u) / u$ is increasing. The proof of uniqueness then follows word-for-word the one given in section 2 .

Finally let us show the claim. If $\lambda(x)>0$ in $\bar{\Omega}$, direct checking reveals that $c$ can be chosen as any positive value such that $u^{p-1}+g(x, u) / u<\inf _{\Omega}(\lambda / a)$ for $x \in \bar{\Omega}$ and $0 \leq u \leq c$. In this case, (14) shows that uniqueness holds provided that $\left(1+M_{g}\right) L_{\Omega}^{2}|a|_{\infty}$ is small. In the case where $\lambda(x)$ changes sign, the argument in Lemma 3.2 of [10] leads to the desired lower estimate for solutions to $(\mathrm{P})_{g}$.

\section{REFERENCES}

1. Bandle C., Essèn M., On the solutions of quasilinear elliptic problems with boundary blow-up, Sympos. Math. 35 (1994), 93-111. MR 95f:35077

2. Bandle C., Marcus M., Sur les solutions maximales de problèmes elliptiques nonlinéaires: bornes isopérimetriques et comportement asymptotique, C. R. Acad. Sci. Paris Série I 311 (1990), 91-93. MR 91f:35096

3. _ 'Large' solutions of semilinear elliptic equations: Existence, uniqueness, and asymptotic behaviour, J. Anal. Math. 58 (1992), 9-24. MR 94c:35081

4. - On second order effects in the boundary behaviour of large solutions of semilinear elliptic problems, Differential Integral Equations 11 (1) (1998), 23-34. MR 98m:35049

5. Bieberbach L., $\Delta u=e^{u}$ und die automorphen Funktionen, Math. Ann. 77 (1916), 173-212.

6. Brézis H., Oswald L., Remarks on sublinear elliptic equations, Nonlinear Anal. 10 (1986), 55-64. MR 87c:35057

7. Del Pino M., Letelier R., The influence of domain geometry in boundary blow-up elliptic problems, Nonlinear Anal. (to appear) (1999).

8. Díaz G., Letelier R., Unicidad de soluciones locales en algunas ecuaciones semilineales, Proceedings of the XIth Congress on Differential Equations and Applications/First Congress on Applied Mathematics, Univ. Málaga, Málaga. (1989), 301-305. MR 91j:00015

9. Díaz G., Letelier R., Explosive solutions of quasilinear elliptic equations: Existence and uniqueness, Nonlinear Anal. 20 (1993), 97-125. MR 94a:35017

10. García-Melián J., Gómez-Reñasco R., López-Gómez J., Sabina de Lis J., Point-wise growth and uniqueness of positive solutions for a class of sublinear elliptic problems where bifurcation from infinity occurs, Arch. Rational Mech. Anal. 145 (1998), 261-289. MR 2000b:35079 
11. D. Gilbarg, N. Trudinger, Elliptic Partial Differential Equations of Second Order, 2nd. edition, Springer Verlag, Berlin/New York, 1983. MR 86c:35035

12. Keller J. B., On solutions of $\Delta u=f(u)$, Comm. Pure Appl. Math. 10 (1957), 503-510. MR 19:964c

13. Kondrat'ev V. A., Nikishkin V. A., Asymptotics, near the boundary, of a solution of a singular boundary value problem for a semilinear elliptic equation, Differential Equations 26 (1990), 345-348. MR 91g:35048

14. Lazer A. C., McKenna P. J., On a problem of Bieberbach and Rademacher, Nonlinear Anal. 21 (1993), 327-335. MR 95b:35070

15. - Asymptotic behaviour of solutions of boundary blow-up problems, Differential Integral Equations 7 (1994), 1001-1019. MR 95c:35084

16. Loewner C., Nirenberg L., Partial differential equations invariant under conformal or projective transformations, Contributions to Analysis (a collection of papers dedicated to Lipman Bers), Academic Press, New York, 1974, p. 245-272. MR 50:10543]

17. Marcus M., Véron L., Uniqueness and asymptotic behaviour of solutions with boundary blowup for a class of nonlinear elliptic equations, Ann. Inst. H. Poincaré Anal. Non Linéaire 14 (2) (1997), 237-274. MR 97m:35068

18. Osserman R., On the inequality $\Delta u \geq f(u)$, Pacific J. Math. 7 (1957), 1641-1647. MR 20:4701

19. Ratto A., Rigoli M., Véron L., Scalar Curvature and Conformal Deformation of Hyperbolic Space, J. Funct. Anal. 121 (1994), 15-77. MR 95a:53062

20. Véron L., Semilinear elliptic equations with uniform blow-up on the boundary, J. Anal. Math. 59 (1992), 231-250. MR 94k:35113

Departamento de Análisis Matemático, Universidad de la laguna, Astrofísico FranCisco SÁnchez S/n, 38271-La Laguna, Spain

E-mail address: jjgarmel@ull.es

Departamento de Matemáticas, Universidad de Concepción, Casilla 3-C, Concepción, CHILE

E-mail address: rletelie@gauss.cfm.udec.cl

Departamento de Análisis Matemático, Universidad de la laguna, Astrofísico FranCisCo SÁNCHez s/N, 38271-LA LAGuna, SPAIN

E-mail address: josabina@ull.es 